\title{
NERO: a pilot study but important step towards comprehensive management of obesity hypoventilation syndrome
}

\author{
Jean-Christian Borel, ${ }^{1,2}$ Anne Laure Borel, ${ }^{1,3}$ Amanda J Piper ${ }^{4}$
}

The primary goal of treatment for obesity hypoventilation syndrome (OHS) has been to correct sleep-related breathing abnormalities, thereby reversing chronic respiratory failure. Nocturnal non-invasive ventilation (NIV) can achieve this and thus represents the cornerstone of respiratory management for this condition. ${ }^{1-4}$ While the effectiveness of NIV in improving clinical symptoms and quality of life has been convincingly demonstrated, ${ }^{235}$ the mortality rate of patients with OHS treated with NIV remains substantially higher than that of patients with obstructive sleep apnoea. ${ }^{6}$ Cardiovascular and metabolic comorbidities are the key determinants of a poorer prognosis. ${ }^{67}$ For several years, clinicians have suggested NIV treatment should be integrated into a comprehensive treatment plan that includes lifestyle modification and rehabilitation to further improve outcomes. ${ }^{8-10}$

In Thorax, Mandal and colleagues ${ }^{11}$ report the first randomised controlled trial evaluating the impact of a 3-month inpatient-outpatient multidisciplinary rehabilitation programme in addition to NIV compared with NIV alone in patients with OHS. Since weight loss is obviously the main treatment for OHS, the primary outcome chosen by the authors was change in weight at 12 months. Although no statistical difference between treatment and control groups was found, this result is clearly inconclusive owing to a lack of statistical power: only 18 patients actually completed the trial while the sample size required was 60 patients. As discussed by the authors, recruitment rate was low and retention at 12 months was poor, with the study being terminated early by the trial data monitoring board. Some of the exclusion criteria could have contributed to this

\footnotetext{
${ }^{1}$ HP2 laboratory (INSERM U1042), Universite Grenoble Alpes, Grenoble, France

${ }^{2}$ Research and Development Department, AGIRà dom, Non Profit Home Care Provider, Meylan, France ${ }^{3}$ Diabetologia and Nutrition Department, Grenoble University Hospital, Grenoble, France

${ }^{4}$ Department of Respiratory and Sleep Medicine, Royal Prince Alfred Hospital, Sydney, New South Wales, Australia
}

Correspondence to Dr Jean-Christian Borel, AGIR à dom Association, 36 bd des Alpes, Meylan 38240, France; j.borel@agiradom.com low recruitment rate: indeed a substantial proportion of patients with OHS may take antipsychotic medication, ${ }^{7}$ have previously experienced acute respiratory failure managed with invasive mechanical ventilation, ${ }^{12}$ or have peripheral artery disease but nevertheless could benefit from a comprehensive rehabilitation programme. Therefore, inclusion/exclusion criteria should be revised in future studies to improve the representativeness of the population of patients with OHS.

The difficulty with recruitment experienced by the authors should not overshadow the strengths and originality of the trial and results. First, although patients with OHS are reputed to be poorly motivated to attend rehabilitation programme, ${ }^{13}$ only $7 \%$ of subjects screened for eligibility declined to participate in the trial, while only two patients $(12 \%)$ allocated to the rehabilitation group withdrew from the programme. The design of the intervention, based on an initial motivational interview followed by personalised diet recommendations and home-based exercise, was probably key to ensuring this good compliance. Furthermore, since nocturnal NIV improves sleep and tends to increase exercise tolerance and sense of well-being, ${ }^{2}{ }^{3}$ the initiation of long-term nocturnal NIV could be the appropriate time to commence a rehabilitation programme in order to reinforce the benefits of therapy, improving motivation and short-term adherence. It has been shown that a lifestyle intervention is less effective in patients with untreated apnoea compared with patients without apnoea, despite similar rate of adherence to the programme. ${ }^{14}$ Indeed, the benefits of combining respiratory treatment with a lifestyle intervention are likely to be bidirectional: lifestyle intervention may improve the respiratory condition as well as significant cardiometabolic comorbidities, while respiratory treatment may increase adherence to, and the success of, the lifestyle intervention programme. Previous studies in patients with hypercapnic COPD have shown that nocturnal NIV combined with a diurnal exercise training programme can enhance the benefits of pulmonary rehabilitation, reduce fatigue and increase daily activity compared with exercise training alone. ${ }^{1516}$

In the current study, the authors also provide the results of several secondary outcomes at 3 months. Although significant $\mathrm{p}$ values should be interpreted with caution due to the possibility of type I errors arising from multiple comparisons, weight loss, exercise capacity and general health perception (as measured by the SF-36) were convincingly improved in the intervention group compared with the control group.

Beyond these functional improvements, arterial blood pressure and waist circumference were also reduced at 3 months. A reduction in waist circumference, which reflects a reduction in visceral adipose tissue, is a major driver for improvement in glucose tolerance and insulin sensitivity. ${ }^{17}$ Although very few studies have specifically assessed the impact of NIV on cardiometabolic markers, none have reported any significant change in these parameters from NIV alone. ${ }^{1-3}$ Therefore, these exploratory results suggest that combining NIV and rehabilitation may attenuate cardiometabolic risks in patients with OHS. Moreover, although the benefits of a rehabilitation programme on body weight are likely to be lost by 12 months, significant long-term cardiometabolic improvements may persist due to the reduction in ectopic fat deposition in liver and muscles. ${ }^{18}$ Thus, in the present study, the long-term aim of the comprehensive rehabilitation programme to reduce body weight could have led to an underestimation of the cardiometabolic benefits of this intervention. Future studies should address the cardiometabolic benefit of such combined interventions by measuring plasma glucose/ insulin homeostasis, lipid and inflammatory profile of patients. ${ }^{19}$

In conclusion, although the results of the (Nutrition and Exercise Rehabilitation in Obesity Hypoventilation Syndrome (NERO) study should be interpreted conservatively because of methodological concerns, this trial has demonstrated that a lifestyle intervention programme that includes exercise and nutrition is feasible for patients with OHS treated with NIV. Moreover, such a programme could reduce body weight and blood pressure, while increasing exercise capacity and health-related quality of life. The results of this pilot study are encouraging with respect to the development of comprehensive treatment programme for OHS, including lifestyle interventions. They also support the establishment of larger multicentre studies in this patient group, with future studies evaluating the long-term effects of these programmes on cardiometabolic and respiratory outcomes. 
Contributors J-CB, ALB and AJP equally contributed to this editorial.

Competing interests None declared.

Provenance and peer review Commissioned; internally peer reviewed.

(c) Article author(s) (or their employer(s) unless otherwise stated in the text of the article) 2018. All rights reserved. No commercial use is permitted unless otherwise expressly granted.

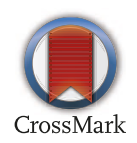

To cite Borel J-C, Borel AL, Piper AJ. Thorax 2018;73:5-6.

Published Online First 26 October 2017

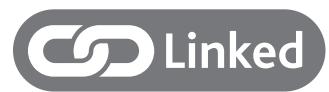

- http://dx.doi.org/10.1136/thoraxjnl-2016-209826

Thorax 2018;73:5-6.

doi:10.1136/thoraxjnl-2017-211032

\section{REFERENCES}

1 Borel JC, Tamisier R, Gonzalez-Bermejo J, et al. Noninvasive ventilation in mild obesity hypoventilation syndrome: a randomized controlled trial. Chest 2012;141:692-702.

2 Masa JF, Corral J, Caballero C, et al. Spanish Sleep Network. Non-invasive ventilation in obesity hypoventilation syndrome without severe obstructive sleep apnoea. Thorax 2016;71:899-906.

3 Masa JF, Corral J, Alonso ML, et al.Efficacy of different treatment alternatives for obesity hypoventilation syndrome. Am J Respir Crit Care Med 2015;192:86-95.

4 Piper AJ, Wang D, Yee BJ, et al. Randomised trial of CPAP vs bilevel support in the treatment of obesity hypoventilation syndrome without severe nocturnal desaturation. Thorax 2008;63:395-401.

5 Murphy PB, Davidson C, Hind MD, et al. Volume targeted versus pressure support non-invasive ventilation in patients with super obesity and chronic respiratory failure: a randomised controlled trial. Thorax 2012;67:727-34.

6 Castro-Añón 0, Pérez de Llano LA, De la Fuente Sánchez S, et al. Obesity-hypoventilation syndrome: increased risk of death over sleep apnea syndrome. PLoS One 2015;10:e0117808.

7 Borel JC, Burel B, Tamisier R, et al. Comorbidities and mortality in hypercapnic obese under domiciliary noninvasive ventilation. PLoS One 2013;8:e52006.

8 Borel JC, Borel AL, Monneret D, et al. Obesity hypoventilation syndrome: from sleep-disordered breathing to systemic comorbidities and the need to offer combined treatment strategies. Respirology 2012;17:601-10.

9 Murphy PB, Polkey MI, Hart N. Obesity hypoventilation syndrome: the need for a multifaceted approach to treatment. Chest 2012;142:540-1.

10 Piper A. Obesity hypoventilation syndrome: weighing in on therapy options. Chest 2016;149:856-68.

11 Mandal S, Suh ES, Harding R, et al. Nutrition and Exercise Rehabilitation in Obesity hypoventilation syndrome (NERO): a pilot randomised controlled trial. Thorax 2018;73:62-9.
12 Carrillo A, Ferrer M, Gonzalez-Diaz G, et al. Noninvasive ventilation in acute hypercapnic respiratory failure caused by obesity hypoventilation syndrome and chronic obstructive pulmonary disease. Am J Respir Crit Care Med 2012;186:1279-85.

13 Jordan KE, Ali M, Shneerson JM. Attitudes of patients towards a hospital-based rehabilitation service for obesity hypoventilation syndrome. Thorax 2009;64:1007.

14 Borel AL, Leblanc X, Alméras N, et al. Sleep apnoea attenuates the effects of a lifestyle intervention programme in men with visceral obesity. Thorax 2012;67:735-41.

15 Duiverman ML, Wempe JB, Bladder G, et al. Nocturnal non-invasive ventilation in addition to rehabilitation in hypercapnic patients with COPD. Thorax 2008;63:1052-7.

16 Garrod R, Mikelsons C, Paul EA, et al. Randomized controlled trial of domiciliary noninvasive positive pressure ventilation and physical training in severe chronic obstructive pulmonary disease. Am J Respir Crit Care Med 2000;162:1335-41.

17 Borel AL, Nazare JA, Smith J, et al. Visceral and not subcutaneous abdominal adiposity reduction drives the benefits of a 1-year lifestyle modification program. Obesity 2012;20:1223-33.

18 Borel AL, Nazare JA, Baillot A, et al. Cardiometabolic risk improvement in response to a 3-yr lifestyle modification program in men: contribution of improved cardiorespiratory fitness vs. weight loss. Am J Physiol Endocrinol Metab 2017;312:E273-81.

19 Chirinos JA, Gurubhagavatula I, Teff K, et al. CPAP, Weight Loss, or Both for Obstructive Sleep Apnea. N Engl J Med Overseas Ed 2014;370:2265-75. 